\title{
COGNITION IN ROBOTS AND ROBOT SCIENTISTS
}

\author{
Soundrarajan.B \\ Department of mechanical engineering \\ THIAGRAJAR COLLEGE OF ENGINEERING, MADURAI-625015
}

\begin{abstract}
The ability of intuition and self- learning in humans is responsible for developing their intelligence, reasoning and socialising. All this human characteristics can enable the robots to evolve into humans. In this context $i$ explain that robots with developing intelligence can solve the problems of various scientific phenomenon such as black-hole, time travels and even in robotics the problems in sensors and actuators which do not impart human level DOF and movement thus making them do everything we can do. Imagine a robot doing yoga, karate, even a ballet all by itself without the rusty old controls and commands. Researchers have come with all kinds of robots and best of all social robots for social interaction so we have come with all kinds of robots what's next? Robot scientists and researchers! Why not? It is highly evident that robot can think in new dimensions to solve issues.
\end{abstract}

\section{KEYWORDS}

Anthropomorphism, tutelage, adaptation, why not? , what if ?, creativity.

\section{INTRODUCTION}

Evolution of the creature's overtime in the world as we know it is because of their tendency to learn from things that they experience. While considering us the human beings we are the greatest achievement nature as given birth .Our ability to adapt and survive come from our way of thinking and accordingly making the best of decisions to compromise in adverse environment. To understand the study of cognitive capability in robots the knowledge of anatomy and functioning of robots is not enough in addition we must also understand emotions and human psychology. In the event of crisis and at the precipice of extinction we humans naturally evolve to better standards and perhaps the cognitive robots and cybernetics will be our next form of evolution.

\section{TALKING TO A TIN CAN IS JUST THE BEGINNING}

The researchers at MIT -BOSTON came with one of the outstanding robot especially for socialising with humans. The Kismet is that robot capable of expressing various facial gestures by perceiving social cues from visual and auditory channel. Kismet has a repository of emotions and only an interaction based robot but when comes to full-fledged free-will robots in addition to facial gestures we might need free-will actions.

Natarajan Meghanathan, et al. (Eds): SIPM, FCST, ITCA, WSE, ACSIT, CS \& IT 06, pp. 585-591, 2012.

(C) CS \& IT-CSCP 2012

DOI : $10.5121 /$ csit.2012.2358 
in robots which are unpredictable. There is one important notion to be considered between social robots and scientist robots, the main difference between the two are the level emotions in them. Looking at the ethology and psychology of humans our progress is always deterred by emotions anger, stress, sorrow, disappointment etc. Obviously these emotions can also reduce their capability so we will be looking at human tin cans that will cry to work.

\section{Emotions affect robots efficiency}

Naturally the robot that can think autonomously and act must have certain restrictions to the negative emotions. It is clearly evident that negative emotions can cause entire failure in the working progress of humans . This must also be applicable to robots, when the robots tend to develop all our capabilities they literally become humans but what is the purpose of having just another metal human competing with worlds gripping increase in population, so the robots must be capable of certain extra perception capabilities that human are not capable of , perhaps robot can create new generation of robots with exceptional thinking, imagination, creativity in entirely new dimensions.So far we have achieved robot that can exhibit anthropomorphism by simply following the human actions and merely imitating them or responding only with facial gestures But here we are dealing with robots that can perform action simultaneously and reasoning for itself whether the action is suitable for the situation or not and what if ? the action fails at that instance thinking a plan for itself to solve in desperate situation. The idea of developing such characteristics is to adapt in the situation so there might not be much difference in the environment in which the technology is acting . The most important thing I would like to remind here is that when we are considering robots we look at an different society of robots socialrobots, artist- robots, worker-robot, player-robot and the most unique of all the are scientist robots.

\section{Our own friendly entertainer}

The social robots are build for the soul purpose to share and interact with similar passion the humans interact with others obviously the emotion level in these robots tops the chart these robots must be build to respond with appropriate gestures in right emotional outbreak In humans Our specimen first gathers data about the users emotional feedback first.

The in-build programming in the robot makes the robot to gather information based on basic questions to the user and should answer why? When? How? Where? But the questioning with reasoning is not needed for robot scientist this why not? The robot then looks into the gathered data about the user and mentions the names and identity of each entity and look up for the users response whether positive or negative, if the response is positive the robot stops if negative it cheers up the user with positive gestures to ease the user, this may be a simple case for the case a social robot when imparting the question why not ? to the social robot, what we may find is a robot that can imagine and reason for itself about everything so this based algorithmic path can enable the robot to be a perfect interactive partner. 


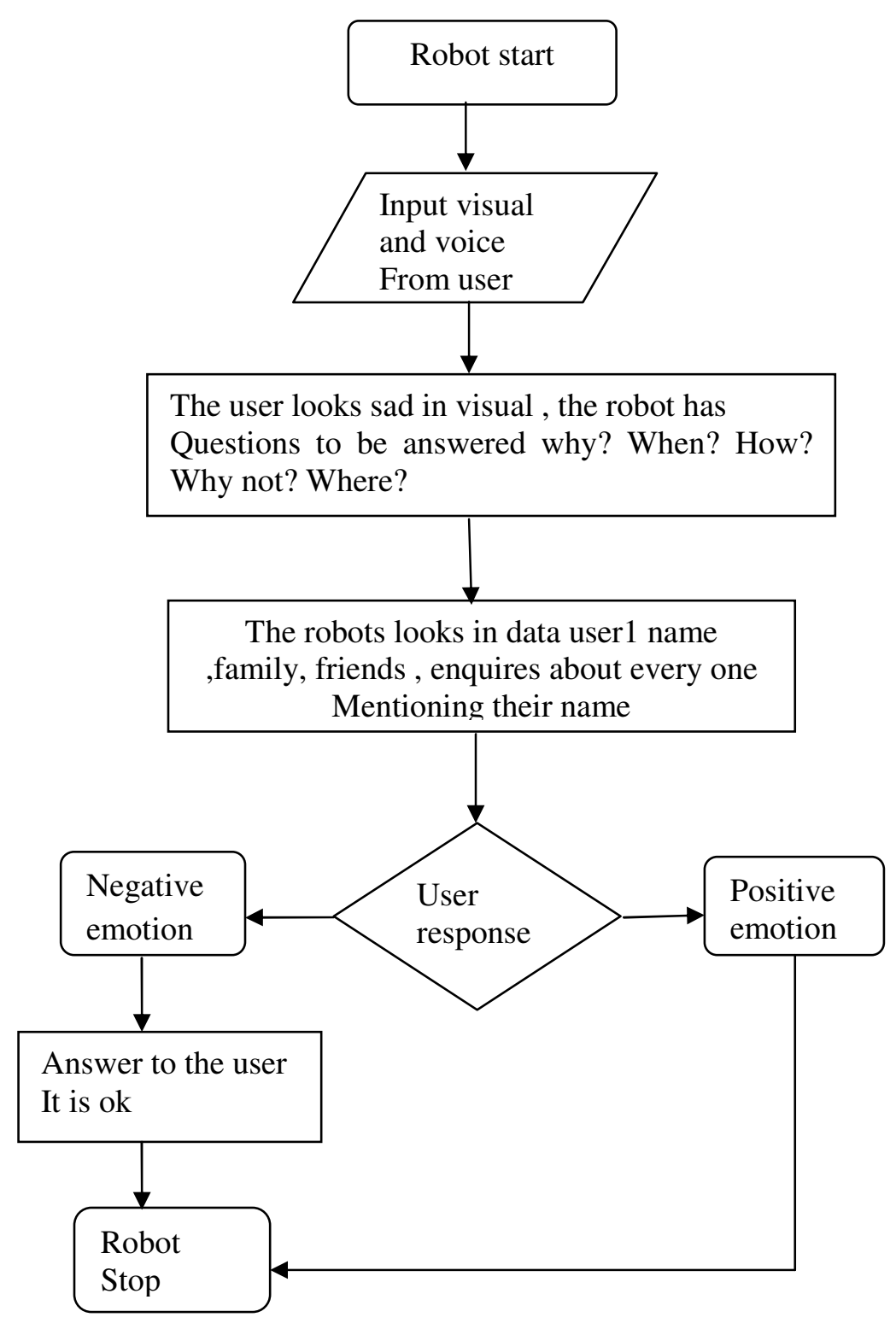

\section{Dawn of robot newtons}

The basic idea that enables human to gather knowledge is to develop interest in a particular field . A typical scientist in making apart from gathering information is to search for ways to answer the questions in a scientific mind.The mother of all technical know -how is to answer why not? commands the robot not only to stop at just finding and gathering informations but also to put them in a comparable manner and seeking for the source and outcomes in basic facts analysing their compatability with one another etc. The notion why not? makes a theory or a hypothesis and what if creates an alternative path for that hypothesis all this enables the robot in lateral way 
of thinking and little advancement can cause even new limitless way thinking capabilities so to make a robot to have autonomous cognitive developments.

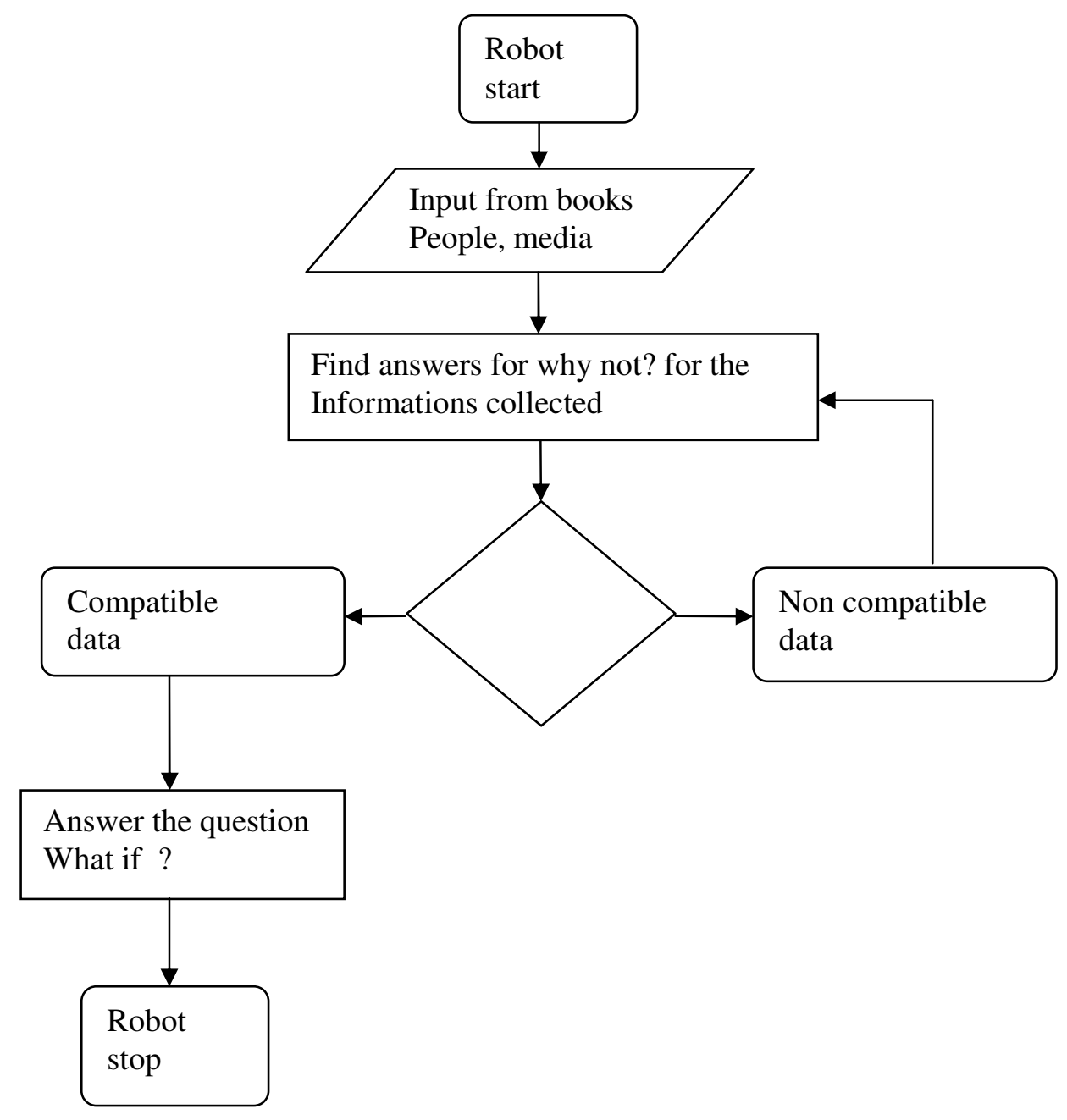

When the robots are limited to simple scientific queries it accepts the existing scientific theories as it is but when breaking the wall to creative thinking that's the instance the robot evolves to natural beings. When we are looking at emotional phenomenon its difficult to explain them in data and numbers then in contextual way.

\section{Limiting of emotions}

We have already discussed about controlling emotions in the robots . Let us put this in this way the robots need not gather negative emotions gathered around them what if ? they can just avoid them by simple programming this is done by a special visual and voice identifier that scans for any negative patterns and does not gathers data.The device is capable of differentiating the emotions and avoiding them so the robot can react only to positive emotions. 

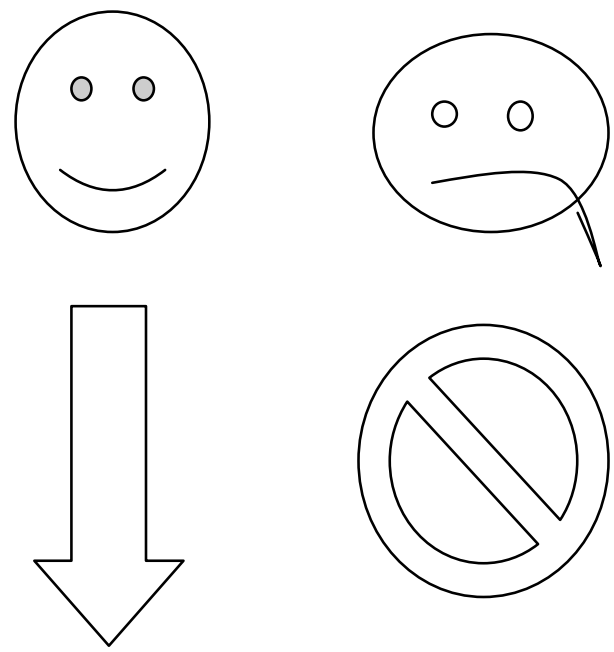

robot

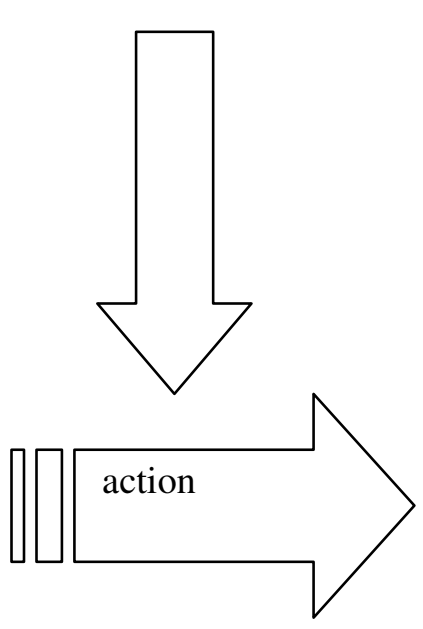

\section{Emotion levels in robots and hierarchy}

There is absolute possibility for robots to have emotions to eventually develop pain and even laziness among themselves the level of cognition can be in increasing from various society of robots this include social robots, scientist robots and professional robots and ground of the system the worker robots the level of cognitive developments goes on in descending order the social robots are pure perfection of humans and can do wrong but are less efficient for tasks ,the scientist robots and the professional robots can have better task efficiency and have creativity as well the last worker robot as the highest task efficiency, but null cognition in them these robots can perform tasks as programmed. The worker robots can be modified to advancements following a method autonomous learning by imitating humans in their actions so called tutelage a simple method like how a child in school learns from the teachers without questioning. The child nevers learns unless it experiences the outcome of the actions. 


\section{Learning from outcomes and environmental response}

The method of learning by gathering data is different from actual experience. The robot takes a rose flower to the user the response of the user to the entity is stored in the robots data base for further reference seeks out answers for the feedback of the user, mentions the names of users that he or she shows negative feedback and determines what entity the negative persons take as positive and concludes that it is not positive for the users, In another case the robot identifies the entity with which the users friends are positive with and identifies it has positive for the user. This type of algorithmic actions in robots make them as service based social robots this gives rise to service robots like nurses, waiters, buttress etc. so any social robots can be of service. The robot that learns before actions is different from the robots that finalizes data before performing the action.

\begin{tabular}{|c|c|c|c|}
\hline robot & thinking & creativity & efficiency \\
\hline social & high & good & poor \\
\hline scientist & high & high & good \\
\hline artist & good & high & fair \\
\hline professional & fair & fair & good \\
\hline service & fair & good & good \\
\hline worker & fair & poor & high \\
\hline humans & high & good & poor \\
\hline
\end{tabular}

\section{Acknowledgements}

It is has been a great pleasure in publishing papers on such interesting field in the science and technology and I thank everyone for training me in depth for the study.

\section{References}

[1] S.Coradeschi, H. Ishiguro, M. Asada, S. Shapiro, M. Theilscher, C. Breazeal, M. Mataric \& H. Ishida .Breazeal and L. Aryananda (2002). "Recognizing affective intent in robot directed speech", Autonomous Robots, 12:1, pp. 83-104.

[2] C.Breazeal and B. Scassellati (2002). "Robots that imitate humans," Trends in Cognitive Science, 6, pp. 481-487.

[3] C.Breazeal, A. Edsinger, P. Fitzpatrick, and B. Scassellati (2001). "Active vision systems for sociable robots," in K. Dautenhahn (ed.), IEEE Transactions on Systems, Man, and Cybernetics, Part A, 31:5, pp. 443-453.

[4] C.Breazeal and B. Scassellati (2000), "Infant-like social interactions between a robot and a human caregiver," in K. Dautenhahn (ed), Adaptive Behavior, 8:1, 47-72.

[5] C.Breazeal, A. Edsinger, P. Fitzpatrick, B. Scassellati (2000), "Social Constraints on Animate Vision," in IEEE Intelligent Systems, Special Issue on Humanoid Robotics, 15:4, 32-37. (2006) Human-Inspired Robots. IEEE Intelligent Systems 21(4), 74-85. 
[6] Soroush Vosoughi and Deb Roy. (2012). A longitudinal study of prosodic exaggeration in childdirected speech. Proceedings of the 6th International Conference on Speech Prosody. Shanghai, China. pdf

[7] Graybiel AM and Kubota Y. (2003) Understanding corticobasal ganglia networks as part of a habit formation system. In: Mental and Behavioral Dysfunction in Movement Disorders, Bedard MA, Agid Y, Chouinard S, Fahn S, Korczyn AD and Lesperance P, Eds. Humana, Totowa NJ

[8] Alvarez, G. A., \& Cavanagh, P. (2004). The capacity of visual short-term memory is set both by visual information load and by number of objects. Psychological Science, 15(2), 106-111.

[9] Common prefrontal regions coactivate with dissociable posterior regions during controlled semantic and phonological tasks. Neuron, 35: 803-812. / Wheeler ME and Buckner RL (2003)

\section{Conclusion}

The event possibility of creating humans in robots through the most advanced method of imparting the cognitive characteristics of humans to robots has been given in the above excerpts in the most simple way and I hope this will pave the way for redefining the autonomous thinking abilities in robots for the sole purpose of serving the science and humanity.

\section{Author}

\section{Soundrarajan.B}

The author is currently pursuing B.E mechanical at thiagrajar college of engineering Madurai and has a keen interest in robotics with cognition, their applications in problem solving for various field of study and admires their capability, in the future.

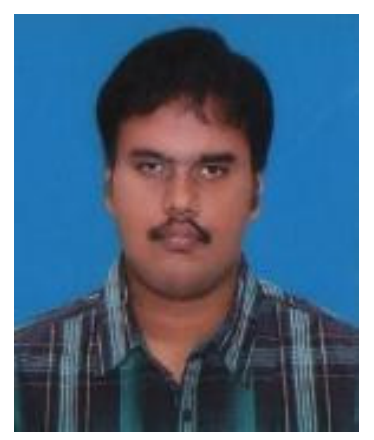

